\title{
Fibonacci Congruences and Applications
}

\author{
René Blacher \\ Laboratory LJK, Université Joseph Fourier, Grenoble, France \\ E-mail: rene.blacher@aliceadsl.fr \\ Received May 24, 2011; revised June 14, 2011; accepted June 20, 2011
}

\begin{abstract}
When we study a congruence $T(x) \equiv a x$ modulo $m$ as pseudo random number generator, there are several means of ensuring the independence of two successive numbers. In this report, we show that the dependence depends on the continued fraction expansion of $m / a$. We deduce that the congruences such that $\mathrm{m}$ and a are two successive elements of Fibonacci sequences are those having the weakest dependence. We will use this result to obtain truly random number sequences $x_{n}$. For that purpose, we will use non-deterministic sequences $y_{n}$. They are transformed using Fibonacci congruences and we will get by this way sequences $x_{n}$. These sequences $x_{n}$ admit the IID model for correct model.
\end{abstract}

Keywords: Fibonacci Sequence, Linear Congruence, Random Numbers, Dependence, Correct Models

\section{Introduction}

In this paper, we present a new method using Fibonacci sequences to obtain real IID sequences $x_{n}$ of random numbers ${ }^{1}$. To have random number two methods exists : 1) use of pseudo-random generators (for example the linear congruence), 2) use of random noise (for example Rap music).

But, up to now no completely reliable solution had been proposed ([1]-[3]). To set straight this situation, Marsaglia has created a Cd-Rom of random numbers by using sequences of numbers provided by Rap music. But, he has not proved that the sequence obtained is really random.

However, by using Fibonacci congruence, there exists simple means of obtaining random sequences whose the quality is sure (cf [4]): one uses the same method as Marsaglia, but one transforms the obtained sequence by Fibonacci congruences. Then, one obtains sequence of real $x_{n}$ such that the IID model is a correct model of $x_{n}$.

\subsection{Fibonacci Congruence}

Linear congruences $T(x) \equiv a x \bmod (\mathrm{m})$ are often used as pseudo-random generators. In this case, we try to choose $\mathrm{a}$ and $\mathrm{m}$ so that successive pseudorandom numbers behave as independent. Of course, we can only ensure that it is the case of $p$ successive numbers $p$ where $2^{p} \leq m$. To choice $a$ and $m$, one can use the spectral test

$\overline{1}$ By abuse of language, we will call "IID sequence" (independent identically distribution) the sequence of random numbers. or the results of Dieter ( $\mathrm{cf}[5])$ which allow to choose the best "a".

Unfortunately, the conditions which ensure the independence of three successive numbers are not those which ensure the best independence of two successive numbers,for example.

Indeed, in this paper, we will study the conditions which ensure the independence of only two successive numbers and we will see with astonishment that this is the Fibonacci congruence which provides the best empirical independence.

We shall study the set

$E_{2}=\{\ell, \overline{T(\ell)} \mid \ell \in\{0,1, \cdots, m-1\}\}$ when $\bar{Z} \equiv z$ modulo $m$ and $0 \leq \bar{Z}<m$ if $z \in \mathbb{Z}$. We will understand that this dependence depends on the continued fraction $\frac{m}{a}$, i.e. it depends on sequences $r_{n}$ and $h_{n}$ defined in the following way.

Notations 1.1 Let $r_{0}=m, r_{1}=a$. One denotes by $r_{n}$ the sequence defined by $r_{n}=h_{n+1} r_{n+1}+r_{n+2}$ the Euclidean division of $r_{n}$ by $r_{n+1}$ when $r_{n+1} \neq 0$. Moreover, one denotes by $d$ the smallest integer such as $r_{d+1}=0$. One sets $r_{d+2}=0$.

One sets $k_{0}=0, k_{1}=1$ and $k_{n+2}=h_{n+1} k_{n+1}+k_{n}$ if $n+1 \leq d$.

Then, dependence depends on the $h_{i}$ 's: more they are small, more the dependence is weak.

Theorem 1 Let $\left(x_{0}, y_{0}\right) \in E_{2}$. Let $R^{0}=\left\{\left[x_{0}, x_{0}+k_{n}\right] \otimes\left[y_{0}, y_{0}+r_{n-2}[\}\right.\right.$ and let $R_{0}=\overline{R^{0}}$, 
be the rectangle $R^{0}$ modulo $m$. Then

If $n$ is even,

$E_{2} \cap R_{0}=\left\{\left(\overline{x_{0}+k_{n-1} \ell}, \overline{y_{0}+r_{n-1} \ell}\right) \mid \ell=0,1,2, \cdots, h_{n-1}\right\} \quad$.

Moreover the points $\left(\overline{x_{0}+k_{n-1} \ell}, \overline{y_{0}+r_{n-1} \ell}\right)$ are lined up modulo $m$.

If $n$ is odd,

$E_{2} \cap R_{0}$

$=\left\{\left(\overline{x_{0}+k_{n-2}+k_{n-1} \ell}, \overline{y_{0}+r_{n-2}-r_{n-1} \ell}\right) \mid \ell=0,1,2, \cdots, h_{n-1}\right\}$.

Moreover, the points $\left(\overline{x_{0}+k_{n-2}+k_{n-1} \ell}, \overline{y_{0}+r_{n-2}-r_{n-1} \ell}\right)$

are lined up modulo $m$.

Of course, in general, it is only on the border that $R_{0}$, the rectangle modulo $m$, satisfies $R_{0} \neq R^{0}$. If not, $R_{0}$ is a normal rectangle.

For example if $x_{0}=y_{0}=0$, this theorem means that the rectangle $\left[0, k_{n} / 2\right] \otimes\left[r_{n-2} / 2, r_{n-2}[\right.$ does not contain points of $E_{2}$ if $n$ is even:

$E_{2} \cap\left\{\left[0, k_{n} / 2\right] \otimes\left[r_{n-2} / 2, r_{n-2}[\}=\varnothing\right.\right.$. If $h_{n-1}$ is large, that will mean that an important rectangle of $\mathbb{R}^{2}$ is empty of points of $E_{2}$ : that will mark a breakdown of independence.

As $h_{i} \geq 1$, the congruence which defines the best independence of $E_{2}$ will satisfy $h_{i}=1$ and $h_{d}=2$. In this case we call it congruence of Fibonacci. Indeed, there exists $n_{0}$ such that $a=f i_{n_{0}}$ and $m=f i_{n_{0}+1}$ where $f i_{n}$ is the sequence of Fibonnacci: $f i_{1}=f i_{2}=1$, $f i_{n+2}=f i_{n+1}+f i_{n}$. As a matter of fact the sequence $r_{n}$ is the sequence of Fibonacci except for the last terms (i.e. except for $\left.f i_{1}=f i_{2}=1\right)$. It is also the case for sequence $k_{n}$.

Remark 1.1 In fact, when we use sequence $h_{n}$, we use Euclidean Algorithm. Now, Dieter has also used this algorithm to compute the dependence of

$\left(T^{n}\left(x_{0}\right), T^{n+1}\left(x_{0}\right)\right)$ when $m=2^{\mathrm{e}}$. But he has not understood the part of the $h_{i}$ 's in this dependence.

\subsection{Application: Building of Random Sequence}

Unfortunately, congruences of Fibonacci cannot be used in order to directly generate good pseudo random sequences because $T^{2}= \pm I d$ where $I d$ is the identity (cf page 141 of [6]). Indeed in this case, the pseudo random sequence $x_{n}=\bar{T}^{n}\left(x_{0}\right)$ checks $x_{n+2}= \pm x_{n}$. However, one can use congruence of Fibonacci in order to build IID sequences by transforming some random noise $y_{n}$.

Definition 1.2 Let $q \in \mathbb{N}^{*}$. Let $T$ be the congruence of Fibonacci modulo $m$. We define the function of Fibonacci $T_{q}$ by $T_{q}=\operatorname{Pr}_{q} \hat{T}$ where

1) $\hat{T}(x)=\bar{T}(m x) / m$,

2) $\operatorname{Pr}_{q}(z)=\overline{\overline{0, b_{1} b_{2} \cdots b_{q}}}$ when $z=\overline{\overline{0, b_{1} b_{2} \cdots}}$ is the binary writing of $z$.

We choose $y_{n} \in\{0 / m, 1 / m, \cdots,(m-1) / m\}, n=1$, $2, \cdots, N$. Then, $y_{n}$ admits for correct model a sequence of random variables $Y_{n}$ defined on a probability space $(\Omega, \mathcal{A}, P)$. Then, we will impose to $y_{n}$ that the conditional probabilities of $Y_{n}$ admit densities with Lipschitz coefficient bounded by $K_{0}$ not too large.

In fact, since $Y_{n}$ has discrete value, we can always assume that $Y_{n}$ has a continuous density.

Notations 1.3 We denote by $\mu_{m}$ be the uniform measure defined on $\{0 / m, 1 / m, \cdots,(m-1) / m\}$ by $\mu_{m}(k / m)=1 / m$ for all $k \in\{0,1, \cdots, m-1\}$.

For all permutation $\phi$ of $\{1,2, \cdots, N\}$, for all $n \in\{1,2, \cdots, N\}$, we denote by $f_{n, \phi}\left(. \mid y_{1}^{\prime}, y_{2}^{\prime}, \cdots\right)$ the conditional density with respect to $\mu_{m}$ of $Y_{\phi(n)}=y$ given $Y_{\phi(n-1)}=y_{1}^{\prime}, Y_{\phi(n-2)}=y_{2}^{\prime}, \cdots$.

Since $Y_{n}$ is discrete, we can also assume that $f_{n, \phi}\left(. \mid y_{1}^{\prime}, y_{2}^{\prime}, \cdots\right)$ has a finite Lipschitz coefficient.

Notations 1.4 We denote by $K_{0}$ a constant such that, for all permutation $\phi$ of $\{1,2, \cdots, N\}$, for all $n \in\{1,2, \cdots, N\}$,

$\left|f_{n, \phi}\left(y \mid y_{1}^{\prime}, y_{2}^{\prime}, \cdots\right)-f_{n, \phi}\left(y^{\prime} \mid y_{1}^{\prime}, y_{2}^{\prime}, \cdots\right)\right| \leq K_{0}\left|y-y^{\prime}\right| . \quad$ In order to simplify the proofs we suppose $K_{0}>1$.

Now, we shall prove easily that the conditional probabilities of $T_{q}\left(Y_{n}\right)$ check

$$
\begin{aligned}
& P\left\{T_{q}\left(Y_{\phi(n)}\right)=x_{0} \mid Y_{\phi(n-1)}=y_{1}^{\prime}, Y_{\phi(n-2)}=y_{2}^{\prime}, \cdots, Y_{\phi(N-1)}=y_{N-1}^{\prime}\right\} \\
& =1 / m\left[1+O(1) K_{0} 2^{q} / m\right]
\end{aligned}
$$

Then we shall choose $m$ and $q$ such that $|\varepsilon| \leq K_{0} 2^{q} / m$ is small enough. We shall deduce that, for all Borel set $B o \subset\left\{0 / 2^{q}, 1 / 2^{q}, \cdots,\left(2^{q}-1\right) / 2^{q}\right\}^{N}$,

$P\left\{\left(X_{1}, \cdots, X_{N}\right) \in B o\right\}=L(B o)[1+O(1) N \varepsilon]$ where $L$ is the measure corresponding to the Borel measure in the case of discrete space : $L\left(\left\{k_{1} / 2^{q}, \cdots, k_{N} / 2^{N}\right\}\right)=1 / 2^{N q}$.

Then $x_{n}=T_{q}\left(y_{n}\right)$ cannot be differentiated from an IID sequence. Indeed, it is wellknown that, for a sample $x_{n}$, there is many models correct : in particular, if $x_{n}$ is extracted of an IID sequence, models such that $P\left\{\left(X_{1}, \cdots, X_{N}\right) \in B o\right\}=L(B o)[1+\varepsilon]$ are correct if $\varepsilon$ is small enough with respect to $\mathrm{N}$. Reciprocally, if the sequence of random variables $X_{n}$ checks

$P\left\{\left(X_{1}, \cdots, X_{N}\right) \in B o\right\}=L(B o)\left[1+\varepsilon_{B o}\right]$, the model IID is also a correct model for the sequence $X_{n}$.

Thus one will be able to admit that the IID model is a correct model for the sequences $x_{n}$. As a matter of fact, one will be even able to admit that there exists another correct model $Y_{n}^{\theta_{0}}$ of $y_{n}$ such that $T_{q}\left(Y_{n}^{\theta_{0}}\right)$ is exactly the IID sequence.

Now there exists noises $y_{n}$ such that $K_{0}$ is not too large. For example these sequences can be built by using texts. In this case we can prove the result : in order that $x_{n}$ is IID, it suffices that $y_{n}$ admits a correct model 
such that $K_{0}$ is not too large. However, it is a condition which can be imposed easily by transforming some noises. The advantage compared with the CD-Rom of Marsaglia is that this result is proved. Of course, we tested such sequences.

So finally we can indeed build sequences $x_{n}$ admitting for correct model the IID model by using Fibonacci congruences. This means that, a priori, these sequences $x_{n}$ behave as random sequences. It is always possible that they do not satisfy certain tests. But it will be a very weak probability as we know it is the case for samples of sequences of IID random variables.

We point out that a first version of these results are in [4]. Moreover, all these results and the proofs are detailed in [7].

Note that to use the congruence of Fibonacci method is completely different from the method using Fibonacci sequence with $X_{n+1}=X_{n}+X_{n-1}$ modulo $\mathrm{m}$, which is moreover a bad generator : cf page 27 of [1].

\section{Dependence Induced by Linear Congruences}

In this section, we study the set $E_{2}=\{\ell, \overline{T(\ell)} \mid \ell \in\{0,1, \cdots, m-1\}\}$ when $T$ is a congruence $T(x) \equiv a x$ modulo $\mathrm{m}$.

\subsection{Notations}

We recall that we define sequences $r_{n}$ and $h_{n}$ by the following way: we set $r_{0}=m, r_{1}=a$ and $r_{n}=h_{n+1} r_{n+1}+r_{n+2}$, the Euclidean division of $r_{n}$ by $r_{n+1}$ when $r_{n+1} \neq 0$. One denotes by $\mathrm{d}$ the smallest integer such as $r_{d+1}=0$. One sets $r_{d+2}=0$. Moreover, $k_{0}=0$, $k_{1}=1$ and $k_{n+2}=h_{n+1} k_{n+1}+k_{n}$ if $n+1 \leq d$.

$$
\text { Then } \frac{m}{a}=h_{1}+\frac{1}{h_{2}+\frac{1}{h_{3}+\frac{1}{h_{4}+\cdots}}} \text {. }
$$

Therefore, $h_{n} \geq 1$ for all $n=1,2, \cdots, d$ and $r_{d-1}=h_{d} r_{d}+r_{d+1}=h_{d} r_{d}+0=h_{d} r_{d}$. The full sequence $r_{n}$ is thus the sequence $r_{0}=m, r_{1}=a, \cdots, r_{d+1}=0$, $r_{d+2}=0$. Then, if $T$ is a Fibonacci conguence, $r_{n}$ is the Fibonacci sequence $f i_{n}$, except for the last terms.

Remark that if $h_{n}=1$ for $n=1,2, \cdots, d-1, k_{n}$ is also the Fibonacci sequence for $n=1,2, \cdots, \mathrm{d}$. Indeed by definition, $k_{0}=0, k_{1}=1$ and $k_{n+2}=h_{n+1} k_{n+1}+k_{n}$ if $n+1 \leq d$.

\subsection{Theorems}

Now, in order to prove the theorem 1, it is enough to prove the following theorem.
Theorem 2 Let $n \in\{2,3, \cdots, d\}$. Then

If $n$ is even,

$E_{2} \cap\left\{\left[0, k_{n}\left[\otimes\left[0, r_{n-2}[\}=\left\{\left(k_{n-1} \ell, r_{n-1} \ell\right) \mid \ell=0,1,2, \cdots, h_{n-1}\right\}\right.\right.\right.\right.$. Moreover the points $\left(k_{n-1} \ell, r_{n-1} \ell\right)$ are lined $u p$.

If $n$ is odd,

$$
\begin{aligned}
& \left.\left.\left.\left.E_{2} \cap\{] 0, k_{n}\right] \otimes\right] 0, r_{n-2}\right]\right\} \\
& =\left\{\left(k_{n-2}+k_{n-1} \ell, r_{n-2}-r_{n-1} \ell\right) \mid \ell=0,1,2, \cdots, h_{n-1}\right\}
\end{aligned} .
$$

Moreover, the points $\left(k_{n-2}+k_{n-1} \ell, r_{n-2}-r_{n-1} \ell\right)$ are lined up.

Then, if there exists $h_{i}$ large, there is a breakdown of independence. For example if $n=2$, it is a wellknown result. Indeed, $m=r_{0}, r_{1}=a, k_{1}=1$ and

$k_{2}=h_{1}=\lfloor m / a\rfloor$ where $\lfloor x\rfloor$ means the integer part of x. Thus, the rectangle Rect $_{2}=[0, m /(2 a)] \otimes[m / 2, m[$ will not contain any point of $E_{2}$. However, this rectangle has its surface equal to $\mathrm{m}^{2} /(4 a)$. Thus if " $a$ " is not sufficiently large, i.e if $h_{1}$ is too large, there is breakdown of independence.

We confirm by graphs the previous conclusion. We suppose $m=21$. If $a=13$, we have a Fibonacci congruence: cf Figure 1. If one chooses $a=10$,

$\sup \left(h_{i}\right)=20: \operatorname{cf}$ Figure 2 . If one chooses $a=5$, $\sup \left(h_{i}\right)=5$ : cf Figure 3 .

Then, in order to avoid any dependence, it is necessary that $\sup \left(h_{i}\right)$ is small.

\subsection{Distribution of $T\left(\left[c, c^{\prime}[)\right.\right.$ When $T$ Is a Fibonacci Congruence}

We assume that $T$ is a Fibonacci congruence. Let $I=\left[c, c^{\prime}\left[\cap\{0,1, \cdots, m-1\}\right.\right.$ where $c, c^{\prime} \in\{0,1, \cdots, m-1\}$. We are interested by $\bar{T}^{-1}(I)$ or $\bar{T}(I)$ because $T^{2}= \pm I d$. Since $\bar{T}(I)$ behaves as independent of $I$, normally, we should find that $\bar{T}(I)$ and, therefore $\bar{T}^{-1}(I)$, is well distributed in $\{0,1, \cdots, m-1\}$. As a matter of fact it is indeed the case.

Indeed, let $k^{n}, n=1,2, \cdots, c^{\prime}-c$, be a permutation of $\left\{c, c+1, \cdots, c^{\prime}-1\right\}$ such that

$\bar{T}^{-1}\left(k^{1}\right)<\bar{T}^{-1}\left(k^{2}\right)<\bar{T}^{-1}\left(k^{3}\right)<\cdots<\bar{T}^{-1}\left(k^{c^{\prime}-c}\right)$. Then,

for all numerical simulations which we executed, one has always obtained

$$
\left|\bar{T}^{-1}\left(k^{r}\right) / m-r / N(I)\right| \leq \varphi(m) / N(I)
$$

where $\varphi(m) \ll \log (m)$. In fact, it seems that $\varphi(m)$ is of the order of $\log (\log (\mathrm{m}))$. Moreover,

$\operatorname{Max}_{r=0,1, \cdots, N(I)-1}\left|N(I) T^{-1}\left(k^{r}\right) / m-r\right|$ seems maximum when I is large enough : $c^{\prime}-c>m / 2$.

For example, in Figures 4, 5 and 6, we have the graphs $N(I) T^{-1}\left(k^{r}\right) / m-r, r=0,1, \cdots, N(I)-1$ for 


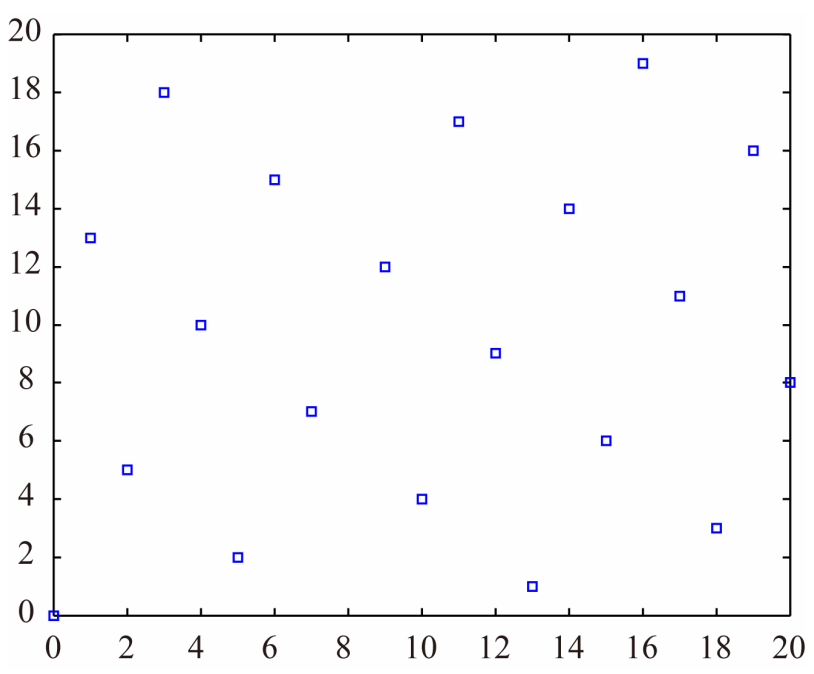

Figure 1. Fibonacci congruence.

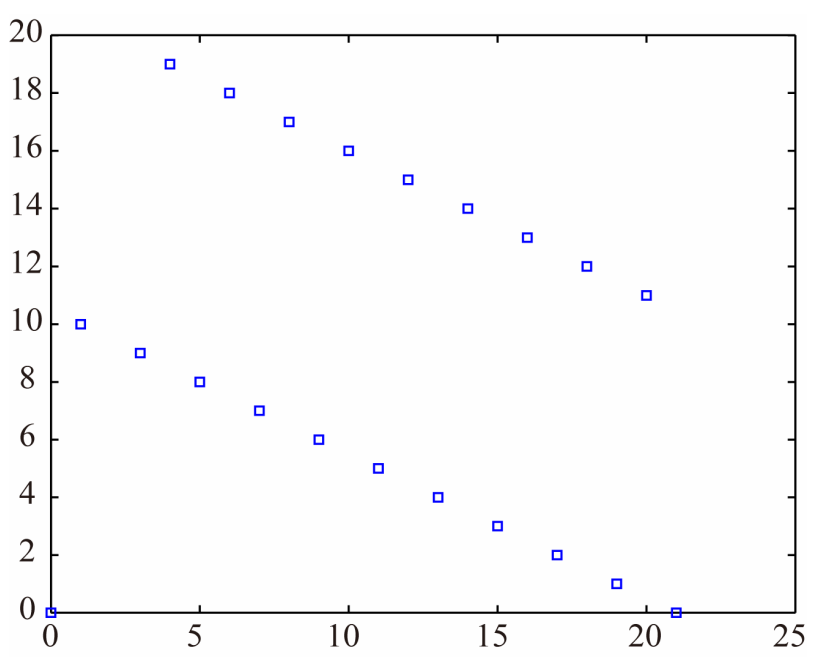

Figure 2. $\sup ($ hi $)=20$.

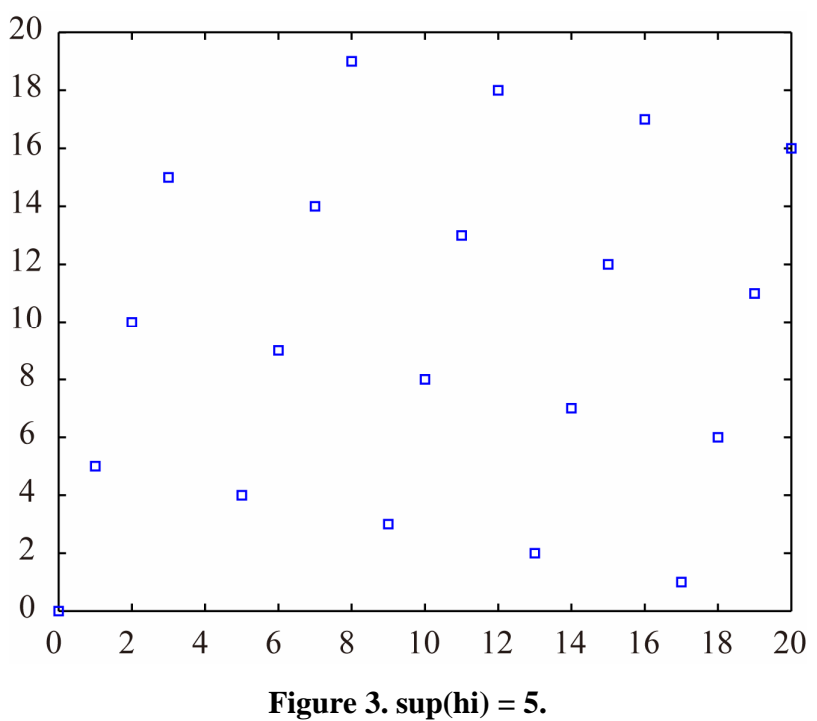

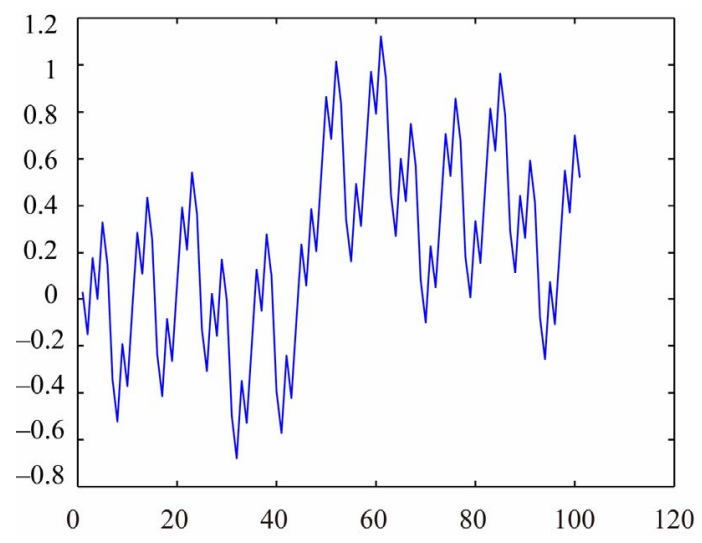

Figure 4. $a=1346269, m=2178309$.

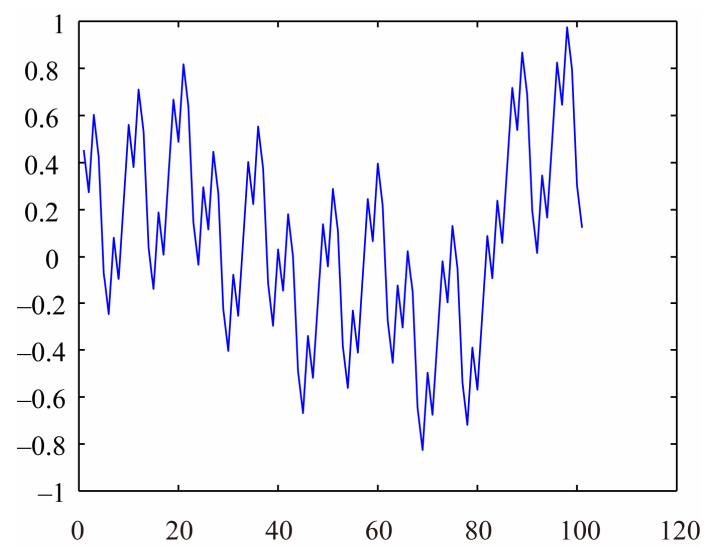

Figure 5. $a=121393, m=196418$.

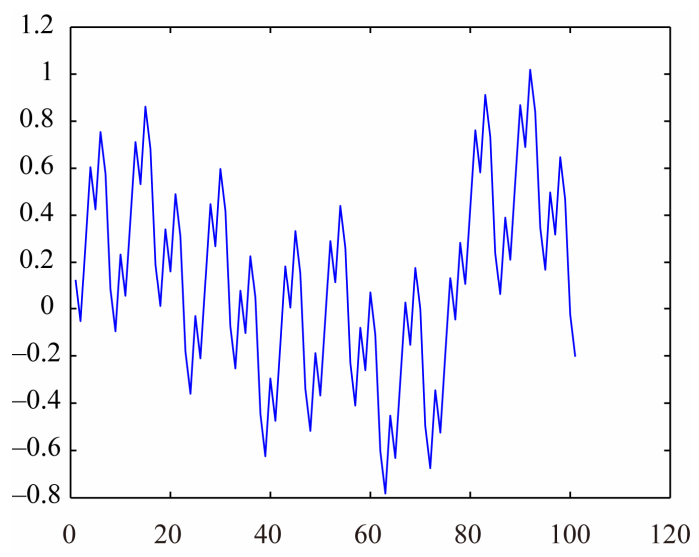

Figure 6. $a=10946, m=17711$.

various Fibonacci congruences when $c^{\prime}-c=100$.

\section{Proof of Theorem 2}

In this section, the congruences are conguences modulo $\mathrm{m}$. Now the first lemma is obvious.

Lemma 3.1 For $n=3,4, \cdots, d+1, k_{n+1}>k_{n}>k_{n-1}$. Moreover $k_{n+2}=h_{n+1} k_{n+1}+k_{n}$ is the Euclidean division 
of $k_{n+2}$ by $k_{n+1}$.

Now, we prove the following results.

Lemma 3.2 Let $n=0,1,2, \cdots, d$. If $n$ is even, $\overline{k_{n} a}=m-r_{n}$. If $n$ is odd, $\overline{k_{n} a}=r_{n}$.

Proof. We prove this lemma by recurrence. For $n=0$,

$\overline{k_{n} a}=\overline{0}=0=m-m=m-r_{0}$. For $n=1$,

$\overline{k_{n} a}=\bar{a}=a=r_{1}$.

We suppose that it is true for $n$.

One supposes $n$ even. Then,

$k_{n+1} a \equiv a h_{n} k_{n}+a k_{n-1} \equiv-h_{n} r_{n}+r_{n-1}=r_{n+1}$.

One supposes $n$ odd. Then,

$k_{n+1} a \equiv a h_{n} k_{n}+a k_{n-1} \equiv h_{n} r_{n}-r_{n-1}=-r_{n+1} \equiv m-r_{n+1}$.

Therefore, $\overline{k_{n+1} a}=m-r_{n+1}$.

Lemma 3.3 Let $n=2,3, \cdots, d+1$. Let

$t \in\left\{1,2, \cdots, k_{n}-1\right\}$. If $n \geq 2$ is even, $r_{n-1} \leq \overline{a t}<m-r_{n}$.

If $n \geq 3$ is odd, $m-r_{n-1} \geq \overline{a t}>r_{n}$.

Moreover, if $n \geq 2$ is even, $\overline{k_{n} a}=m-r_{n}$. If $n \geq 3$ is odd, $\overline{k_{n} a}=r_{n}$.

Proof. The second assertion is lemma 3.2. Now, we prove the first assertion by recurrence.

One supposes $n=2$. Then, $m=r_{0}=h_{1} r_{1}+r_{2}=h_{1} a+r_{2}$. Moreover, $k_{2}=h_{1}$. If $1 \leq t<h_{1}=k_{2}$, $r_{1}=a \leq a t<h_{1} a=m-r_{2}$.

If $h_{1}=k_{2}=1,\left\{1,2, \cdots, k_{2}-1\right\}=\varnothing$. In this case, we study $t \in\left\{1,2, \cdots, k_{3}-1\right\}$ where $k_{3}=h_{2} k_{2}+k_{1}=h_{2}+1$. Then, $1 \leq t \leq h_{2}$. Then, at $\equiv t a k_{2} \equiv-t r_{2}$.

Moreover,

$$
\begin{aligned}
& m-r_{2} \geq m-t r_{2} \geq m-h_{2} r_{2}=r_{0}-h_{2} r_{2} \\
& =r_{0}-\left(r_{1}-r_{3}\right)=r_{3}+\left(r_{0}-r_{1}\right)>r_{3}
\end{aligned}
$$

Therefore, because $a t \equiv m-t r_{2}, \overline{a t}=m-t r_{2}$.

Therefore, $m-r_{2} \geq \overline{a t}>r_{3}$.

One supposes that the first assertion is true for $n$ where $2 \leq n \leq d$.

Let $0<t^{\prime}<k_{n+1}$. Let $t^{\prime}=f k_{n}+e$ be the Euclidean division of $t^{\prime}$ by $k_{n}: e<k_{n}$.

Then, $f \leq h_{n}$. If not,

$t^{\prime} \geq\left(h_{n}+1\right) k_{n}+e \geq h_{n} k_{n}+k_{n-1}=k_{n+1}$.

One supposes $n$ even.

In this case, $r_{n-1} \leq \overline{a t}<m-r_{n}$ for

$t \in\left\{1,2, \cdots, k_{n}-1\right\}$.

Moreover,

$a t^{\prime} \equiv f a k_{n}+a e \equiv f\left(m-r_{n}\right)+a e \equiv-f r_{n}+a e$.

First, one supposes $e=0$. Then, $f \geq 1$.

Moreover, because $n \geq 2$,

$m-r_{n} \geq m-f r_{n} \geq m-h_{n} r_{n}=m-\left(r_{n-1}-r_{n+1}\right)$.

$=r_{0}-r_{n-1}+r_{n+1} \geq r_{0}-r_{1}+r_{n+1}>r_{n+1}$

Therefore, because $a t^{\prime} \equiv-f r_{n}, \overline{a t^{\prime}}=m-f r_{n}$.

Therefore, $m-r_{n} \geq \overline{a t^{\prime}}>r_{n+1}$.

Now, one supposes $f<h_{n}$ and $e>0$.
By recurrence,

$m-r_{n} \geq \overline{a e} \geq \overline{a e}-f r_{n} \geq r_{n-1}-f r_{n} \geq r_{n-1}-\left(h_{n}-1\right) r_{n}$.

$=r_{n}+r_{n+1}>r_{n+1}$

Therefore, because $a t^{\prime} \equiv-f r_{n}+a e, \overline{a t^{\prime}}=\overline{a e}-f r_{n}$.

Therefore, $m-r_{n} \geq \overline{a t^{\prime}}>r_{n+1}$.

One supposes $f=h_{n}, e \neq k_{n-1}$ and $e>0$.

If $e \neq k_{n-1}, \overline{a e} \neq \overline{k_{n-1} a}$. Indeed, if not,

$\overline{a\left(e-k_{n-1}\right)}=0$. For example, if $e-k_{n-1}>0$,

$k_{n}>e-k_{n-1}>0$. Then, because our recurence,

$\overline{a\left(e-k_{n-1}\right)}>r_{n-1}>0:$ it is impossible.

Now, if $n=2, \overline{k_{n-1} a}=\overline{k_{1} a}=\bar{a}=r_{1}=r_{n-1}$.

Moreover, if $n>2, n \geq 4$. Then, by recurence $\overline{k_{n-1} a}=r_{n-1}$.

Then, if $e \neq k_{n-1}, \overline{a e} \neq \overline{k_{n-1} a}=r_{n-1}$. Then, $\overline{a e}>r_{n-1}$.

Moreover,

$m-r_{n} \geq \overline{a e} \geq \overline{a e}-f r_{n}>r_{n-1}-f r_{n} \geq r_{n-1}-h_{n} r_{n}=r_{n+1}$.

Therefore, because $a t^{\prime} \equiv-f r_{n}+a e, \overline{a t^{\prime}}=\overline{a e}-f r_{n}$.

Therefore, $m-r_{n} \geq \overline{a t^{\prime}}>r_{n+1}$.

One supposes $f=h_{n}$ and $e=k_{n-1}$. Then,

$t^{\prime}=h_{n} k_{n}+k_{n-1}=k_{n+1}$. It is opposite to the assumption.

Then, in all the cases, for $t^{\prime} \in\left\{1,2, \cdots, k_{n+1}-1\right\}$, $m-r_{n} \geq \overline{a t^{\prime}}>r_{n+1}$. Therefore, the lemma is true for $n+1$ if $n$ is even. Then, it is also true for $n+1=3$.

One supposes $n$ odd with $n \geq 3$. One proves the recurrence by the same way as if $n$ is even $(\operatorname{cf}[7])$. Then the lemma is true for $\mathrm{n}+1$.

Lemma 3.4 The following inequalities holds: $k_{d+1} \leq m$.

Proof. If $t \in\left\{1,2, \cdots, k_{d+1}-1\right\}$, by lemma 3.3, $r_{d} \leq \overline{a t}<m-r_{d+1}$ or $m-r_{d} \geq \overline{a t}>r_{d+1}$, i.e. $r_{d} \leq \overline{a t}<m$ or $m-r_{d} \geq \overline{a t}>0$ where $r_{d}>0$. Then, $0<\overline{a t}<m$ or $m>\overline{a t}>0$.

Then, if $k_{d+1}>m$, there exists $t_{0} \in\left\{1,2, \cdots, k_{d+1}-1\right\}$ such that $t_{0}=m$, i.e. $\overline{a t_{0}}=\overline{a m}=0$. It is impossible.

Lemma 3.5 Let $t, t^{\prime} \in\left\{1,2, \cdots, k_{d+1}-1\right\}$ such that $\overline{a t}=\overline{a t^{\prime}}$. Then, $t=t^{\prime}$.

Proof. Suppose $t>t^{\prime}$. Then, $a\left(t-t^{\prime}\right) \equiv 0$ and $\overline{a\left(t-t^{\prime}\right)}=0$. Then, by lemma 3.3, $r_{d} \leq \overline{a\left(t-t^{\prime}\right)}<m-r_{d+1}$ or $m-r_{d} \geq \overline{a\left(t-t^{\prime}\right)}>r_{d+1}=0$ where $r_{d}>0$. Then, $0<\overline{a\left(t-t^{\prime}\right)}$. It is a contradiction.

Lemma 3.6 Let $n=1,2, \cdots, d$. Let

$H_{n}=h_{1} k_{1}+h_{2} k_{2}+h_{3} k_{3}+\cdots+h_{n} k_{n}$. Then, $H_{n}=k_{n+1}+k_{n}-1$

The proof is basic.

Lemma 3.7 Let $n=1,2,3, \ldots, d-1$. Let $L_{n}=\left\{t \mid t=0,1,2, \cdots, H_{n}\right\}$. Then, for all $n \geq 1$, $L_{n+1}=\left\{t=l+g k_{n+1} \mid l \in L_{n}, g \leq h_{n+1}\right\}$. 
Proof. Let $l \in L_{n}, l \leq H_{n}$. Let $g \leq h_{n+1}$. Therefore, if $t=l+g k_{n+1}, t \leq H_{n}+h_{n+1} k_{n+1}=H_{n+1}$. Therefore, $\left\{t=l+g k_{n+1} \mid l \in L_{n}, g \leq h_{n+1}\right\} \subset L_{n+1}$.

Reciprocally, let $t \in L_{n+1}$ and let $t=f k_{n+1}+e$, $e<k_{n+1}$ be the Euclidean division of t by $k_{n+1}$.

We know that $H_{n}=k_{n+1}+k_{n}-1 \geq k_{n+1}$. Therefore, $e \leq H_{n}$. Therefore, $e \in L_{n}$.

Therefore, if $f \leq h_{n+1}$, $t=f k_{n+1}+e \in\left\{t=l+g k_{n+1} \mid l \in L_{n}, g \leq h_{n+1}\right\}$.

Moreover, if $f>h_{n+1}+1$,

$t=f k_{n+1}+e \geq\left(h_{n+1}+2\right) k_{n+1}+e \geq h_{n+1} k_{n+1}+2 k_{n+1}$

$=H_{n+1}-H_{n}+2 k_{n+1}=H_{n+1}-k_{n+1}-k_{n}+1+2 k_{n+1}$.

$=H_{n+1}+k_{n+1}-k_{n}+1 \geq H_{n+1}+1$

Therefore, $t \notin L_{n+1}$.

Then, suppose $f=h_{n+1}+1$. Then,

$t=f k_{n+1}+e=\left(h_{n+1}+1\right) k_{n+1}+e$

$=h_{n+1} k_{n+1}+k_{n+1}+e=H_{n+1}-H_{n}+k_{n+1}+e$

$=H_{n+1}-k_{n+1}-k_{n}+1+k_{n+1}+e=H_{n+1}-k_{n}+1+e$

Because $t \in L_{n+1}$ and $t=H_{n+1}-k_{n}+1+e$, $e+1-k_{n} \leq 0$. Therefore, $e \leq k_{n}-1$.

Therefore, $t=f k_{n+1}+e=h_{n+1} k_{n+1}+k_{n+1}+e$,

where $k_{n+1}+e \leq k_{n+1}+k_{n}-1=H_{n}$

Therefore, $t=h_{n+1} k_{n+1}+e^{\prime}$ where $e^{\prime} \leq H_{n}$.

Therefore, $t \in\left\{t=l+g k_{n+1} \mid l \in L_{n}, g \leq h_{n+1}\right\}$.

Therefore, $L_{n+1} \subset\left\{t=l+g k_{n+1} \mid l \in L_{n}, g \leq h_{n+1}\right\}$.

Therefore, $L_{n+1}=\left\{t=l+g k_{n+1} \mid l \in L_{n}, g \leq h_{n+1}\right\}$.

Lemma 3.8 Let $F_{n}=\left\{\overline{a t} \mid t=0,1,2, \cdots, H_{n}\right\}$.

Let $E_{n}=\left\{\overline{a t}+k m \mid t=0,1,2, \cdots, H_{n}, k \in Z\right\}$. We set

$E_{n}=\left\{o_{s}^{n} \mid s \in Z\right\}$ where $o_{0}^{n}=0$ and $o_{s+1}^{n}>o_{s}^{n}$ for all $s \in Z$.

Then, for all $s \in Z, \quad o_{s+1}^{n}-o_{s}^{n}=r_{n}$ or $o_{s+1}^{n}-o_{s}^{n}=r_{n+1}$.

Proof We prove this lemma by recurrence.

Suppose $n=1$. Then, $r_{1}=a, H_{1}=h_{1} k_{1}=k_{2}=h_{1}$. Therefore,

$$
\begin{aligned}
& F_{1}=\left\{\overline{a t} \mid t=0,1,2, \cdots, h_{1}\right\}=\left\{0, a, 2 a, \cdots, h_{1} a\right\} . \\
& =\left\{0, r_{1}, 2 r_{1}, \cdots, h_{1} r_{1}=m-r_{2}\right\}
\end{aligned}
$$

Therefore, the lemma is true for $n=1$.

Suppose that the lemma is true for $n$.

Then, $E_{n+1}=\left\{\overline{a t}+k m \mid t=0,1,2, \cdots, H_{n+1}, k \in Z\right\}$, where

$$
H_{n+1}=h_{1} k_{1}+h_{2} k_{2}+h_{3} k_{3}+\cdots+h_{n+1} k_{n+1}=H_{n}+h_{n+1} k_{n+1} \text {. }
$$

Because $t \in\left\{0,1,2, \cdots, H_{n+1}\right\}, t \in L_{n+1}$. By lemma 3.7, If $t \in L_{n+1}, t=l+g k_{n+1}$ where $g \leq h_{n+1}$. By lemma 3.2, $\overline{a t} \equiv \overline{a\left(l+g k_{n+1}\right)} \equiv \overline{a l}+(-1)^{n+2} g r_{n+1} \equiv \overline{a l}+(-1)^{n} g r_{n+1}$.
Therefore,

$$
\begin{aligned}
& E_{n+1}=\left\{\overline{a t}+k m \mid t \in L_{n+1}, k \in Z\right\} \\
& =\left\{\overline{a t}+k m \mid t=l+g k_{n+1}, l \in L_{n}, g \leq h_{n+1}, k \in Z\right\} \\
& =\left\{\overline{a l}+(-1)^{n} g r_{n+1}+k m \mid l \in L_{n}, g \leq h_{n+1}, k \in Z\right\} \\
& =\left\{f+(-1)^{n} g r_{n+1}+k m \mid f \in F_{n}, g \leq h_{n+1}, k \in Z\right\} \\
& =\left\{o_{s}^{n}+(-1)^{n} g r_{n+1}+k m \mid s \in Z, g \leq h_{n+1}, k \in Z\right\}
\end{aligned}
$$

Suppose that $n$ is even.

Then, $o_{s}^{n}+(-1)^{n} g r_{n+1}=o_{s}^{n}+g r_{n+1} \leq o_{s}^{n}+r_{n}-r_{n+2}$

because $g r_{n+1} \leq h_{n+1} r_{n+1}=r_{n}-r_{n+2}$.

Use the recurrence. Suppose $o_{s+1}^{n}-o_{s}^{n}=r_{n}$. Then, $o_{s}^{n}+(-1)^{n} g r_{n+1} \leq o_{s}^{n}+r_{n}-r_{n+2}=o_{s+1}^{n}-r_{n+2}$.

Therefore,

$\left\{o_{t}^{n+1} \mid o_{s}^{n} \leq o_{t}^{n+1}<o_{s+1}^{n}\right\}$

$=\left\{o_{s}^{n}<o_{s}^{n}+r_{n+1}<\cdots<o_{s}^{n}+h_{n+1} r_{n+1}<o_{s+1}^{n}\right\}$

Therefore, $o_{t+1}^{n+1}-o_{t}^{n+1}=r_{n+1}$ or $r_{n+2}$ if $o_{s}^{n} \leq o_{t}^{n+1}<o_{t+1}^{n+1} \leq o_{s+1}^{n}$.

Suppose $o_{s+1}^{n}-o_{s}^{n}=r_{n+1}$. Then, $s$ is fixed.

Let $T=\min \left\{t=0,1, \cdots, \mid o_{s+t+1}^{n}-o_{s+t}^{n}=r_{n}\right\}$. Therefore, $o_{s+T+1}^{n}-o_{s+T}^{n}=r_{n}$.

Let $O=\cup_{t=0}^{T}\left\{o_{s+t}^{n}+g r_{n+1} \mid 0 \leq g \leq h_{n+1}\right\}$.

Then,

$O=\left\{o_{s}^{n}, o_{s+1}^{n}, \cdots, o_{s+T-1}^{n}\right\} \cup\left\{o_{s+T}^{n}+g r_{n+1} \mid 0 \leq g \leq h_{n+1}\right\}$.

Therefore, $O=\left\{o_{s}^{\prime}, o_{s+1}^{\prime}, \cdots, o_{s+K}^{\prime}\right\}$ where $o_{s^{\prime}+1}^{\prime}-o_{s^{\prime}}^{\prime}=r_{n+1}$. Moreover,

$o_{s+T+1}^{n}-o_{s+K}^{\prime}=r_{n}-h_{n+1} r_{n+1}=r_{n+2}$.

Therefore, if $O_{t^{\prime}+1}^{n+1}$ and $o_{t^{\prime}+1}^{n+1} \in\left\{o_{t}^{n+1} \mid o_{s}^{n} \leq o_{t}^{n+1} \leq o_{s+T+1}^{n}\right\}, \quad o_{t^{\prime}+1}^{n+1}-o_{t^{\prime}}^{n+1}=r_{n+1}$ or $r_{n+2}$.

Suppose that $n$ is odd. One proves this result by the same way as when $\mathrm{n}$ is even (cf [7]).

Proof 3.9 Now one proves theorem 2.

Suppose that $n$ is even.

Then, $\overline{k_{n-1} a}=r_{n-1}, \quad 2 \overline{k_{n-1} a}=2 r_{n-1}, \ldots \ldots$.

$h_{n-1} \overline{k_{n-1} a}=h_{n-1} r_{n-1}=r_{n}-r_{n-2}$.

Now, $\overline{a k_{n-1} \ell}=\overline{\ell r_{n-1}}=\ell r_{n-1}$ for $\ell=0,1,2, \cdots, h_{n-1}$.

$\left\{\left(k_{n-1} \ell, r_{n-1} \ell\right) \mid \ell=0,1,2, \cdots, h_{n-1}\right\}$

$=\left\{\left(k_{n-1} \ell, \overline{a k_{n-1} \ell}\right) \mid \ell=0,1,2, \cdots, h_{n-1}\right\} \subset E_{2}$ Therefore,

Moreover, $r_{n-2}=h_{n-1} r_{n-1}+r_{n}$. On the other hand, by lemma 3.8 , all the points of $E_{2}=(t, \overline{a t}), t \leq H_{n-1}$, have ordinates distant of $r_{n}$ or $r_{n-1}$.

Therefore, if there is other points of $E_{2} \cap\left\{\left[0, H_{n-1}\right] \otimes\left[0, r_{n-2}[\}\right.\right.$ that the points $\left\{\left(k_{n-1} \ell, r_{n-1} \ell\right) \mid \ell=0,1,2, \cdots, h_{n-1}\right\}$, there exists $\ell_{0} \in\left\{1,2, \cdots, h_{n-1}\right\}$ and 
$\left(x_{1}, y_{1}\right) \in E_{2} \cap\left\{\left[0, H_{n-1}\right] \otimes\left[0, r_{n-2}\right]\right\}$ such that $r_{n-1} \ell_{0}-y_{1}=r_{n}$

Because $H_{n-1}=k_{n}+k_{n-1}-1<k_{n+1} \leq k_{d+1}$, by lemma 3.5, there exists an only $t \in\left\{1, \cdots, H_{n-1}\right\}$, such that $\overline{a t}=y_{1}: t=x_{1}$. Because $y_{1} \neq 0$, there exists an only $t \in\left\{0,1, \cdots, H_{n-1}\right\}$, such that $\overline{a t}=y_{1}$.

Now, $\quad r_{n-1} \ell_{0}-y_{1}=\overline{a \ell_{0} k_{n-1}}-\overline{a t}=r_{n}=\overline{-a k_{n}}$. Then, $\overline{a \ell_{0} k_{n-1}}-\overline{-a k_{n}}=\overline{a t}$. Then, $\overline{a\left(\ell_{0} k_{n-1}+k_{n}\right)}=\overline{a t}$.

Because $\quad r_{d-1}=h_{d} r_{d}$ with $r_{d-1}>r_{d}, h_{d} \geq 2$. Moreover, $d \geq n \geq 2$. Then, $d-1>0$. Then, $k_{d-1}>0$.

Then, by lemma 3.4 ,

$2 k_{n}-k_{n-2} \leq 2 k_{d}<2 k_{d}+k_{d-1} \leq h_{d} k_{d}+k_{d-1}=k_{d+1} \leq m$.

Then, $0<\ell_{0} k_{n-1}+k_{n}<k_{d+1}$.

Then, by lemma 3.4 ,

$0<k_{n-1}+k_{n} \leq \ell_{0} k_{n-1}+k_{n} \leq h_{n-1} k_{n-1}+k_{n} \leq k_{n}-k_{n-2}+k_{n}$

$=2 k_{n}-k_{n-2} \leq 2 k_{d}<2 k_{d}+k_{d-1} \leq h_{d} k_{d}+k_{d-1}=k_{d+1} \leq m$

Then, $0<\ell_{0} k_{n-1}+k_{n}<k_{d+1}$.

Now $0<t \leq H_{n-1}=k_{n}+k_{n-1}-1<k_{d}+k_{d-1} \leq k_{d+1}$.

Moreover, $0<\ell_{0} k_{n-1}+k_{n}<k_{d+1}$.

Then, because $\overline{a\left(\ell_{0} k_{n-1}+k_{n}\right)}=\overline{a t}$, by lemma 3.5, $t=\ell_{0} k_{n-1}+k_{n}$.

Then, $\quad t=\ell_{0} k_{n-1}+k_{n} \geq k_{n-1}+k_{n}>H_{n-1}$. It is a contradiction.

Therefore, there is not other points of

$E_{2} \cap\left\{\left[0, H_{n-1}\right] \otimes\left[0, r_{n-2}[\}\right.\right.$ that

$\left\{\left(k_{n-1} \ell, r_{n-1} \ell\right) \mid \ell=0,1,2, \cdots, h_{n-1}\right\}$.

Therefore, there is not other points of $E_{2} \cap\left\{\left[0, k_{n}\left[\otimes\left[0, r_{n-2}[\}\right.\right.\right.\right.$ that the points $\left\{\left(k_{n-1} \ell, r_{n-1} \ell\right) \mid \ell=0,1,2, \cdots, h_{n-1}\right\}$.

Therefore,

$E_{2} \cap\left\{\left[0, k_{n}\left[\otimes\left[0, r_{n-2}[\}=\left\{\left(k_{n-1} \ell, r_{n-1} \ell\right) \mid \ell=0,1,2, \cdots, h_{n-1}\right\}\right.\right.\right.\right.$.

According to what precedes,

$\left\{\left(k_{n-1} \ell, \overline{a k_{n-1} \ell}\right) \mid \ell=0,1,2, \cdots, h_{n-1}\right\}$

$=\left\{\left(k_{n-1} \ell, r_{n-1} \ell\right) \mid \ell=0,1,2, \cdots, h_{n-1}\right\}$

is located on the straight line $y=\left(r_{n-1} / k_{n-1}\right) x$ if $n$ is even.

Suppose that $n$ is odd. One proves this result by the same way as when $n$ is even (cf [7]).

\section{Models Equivalent with a Margin of $\varepsilon$}

\subsection{Correct Models}

In general terms, one can always suppose that $y_{n}$ is the realization of a sequence of random variables $Y_{n}$ defined on a probability space $(\Omega, A, P): y_{n}=Y_{n}(\omega)$ where $\omega \in \Omega$ and where $Y_{n}$ is a correct model of $y_{n}$.
As a matter of fact, there exist an infinity of correct models of $y_{n}$. It is thus necessary to be placed in the set of all the possible random variables.

Notations 4.1 One considers the sequences of random variables $Y_{n}^{\theta}, n=1, \cdots, N$, defined on the probabilities spaces $\left(\Omega, A, P_{\theta}\right), \quad \theta \in \Theta$ :

$\left(Y_{1}^{\theta}, Y_{2}^{\theta}, \cdots, Y_{N}^{\theta}\right): \Omega \rightarrow\{0 / m, 1 / m, \cdots,(m-1) / m\}^{N}$. One assumes that $Y_{n}^{\theta}=Y_{n}$ for all $\theta \in \Theta$.

For example, one can assume that $\Omega=\{0 / m, 1 / m, \cdots,(m-1) / m\}^{N}$ and $\left(Y_{1}, \cdots, Y_{N}\right)=($ Id $, \cdots, I d)$.

It thus raises the question to define what is a correct model. Indeed, if a model $Y_{n}^{\theta}$ is not correct, it is however possible that $y_{n}=Y_{n}^{\theta}(\omega)$. Now, in the case where the model $Y_{n}^{\theta}$ is IID, to define a correct model is a generalization of the problem of the definition of an IID sequence. Then, it is a very complex problem (cf [1]).

However, generally, one feels well that correct models exist. In fact, it is a traditional assumption in science. In weather for example, the researchers seek a correct model, which implies its existence (if not, why to try to make forecasts?).

One could thus admit that like a conjecture or a postulate without defining exactly what is a correct model. Cependant, a more detailed study is in [7].

\subsection{Models Equivalent}

\subsubsection{The Problem}

Let $Y_{n}^{\theta_{2}}$ and $Y_{n}^{\theta_{1}}$ be two sequences of random variables such that, for all Borel set Bo,

$P\left\{\left(Y_{1}^{\theta_{2}}, \cdots, Y_{N}^{\theta_{2}}\right) \in B o\right\}=P\left\{\left(Y_{1}^{\theta_{1}}, \cdots, Y_{N}^{\theta_{1}}\right) \in B o\right\}[1+O b(1) \varepsilon]$,

where $\mathrm{Ob}($.$) means the classical \mathrm{O}($.$) with the additional$ condition $|O b(1)| \leq 1$. One supposes that $Y_{n}^{\theta_{1}}$ is a correct model of the sequence $y_{n}, n=1,2, \cdots, N$. One wants to prove that $Y_{n}^{\theta_{2}}$ is also a correct model of $y_{n}$ if $\varepsilon$ is small enough.

\subsubsection{Example}

Let us suppose that we have a really IID sequence of random variables $X_{n}^{\varepsilon}$ with uniform distribution on $[0,1 / 2]$ and $[1 / 2,1]$ and with a probability such as $P\left\{X_{n}^{\varepsilon} \in[1 / 2,1]\right\}=0,500[1+\varepsilon] \quad$ where $\quad \varepsilon=0,001$. Then, this sequence has not the uniform distribution on $[0,1]$. However, if we have a sample with size 10 , we will absoluetely not understand that $X_{n}^{\varepsilon}$ has not the uniform distribution on $[0,1]$. It is wellknown that one need samples with size larger than $\mathrm{N}=1000$ minimum in order to test this difference.

For example, one cannot test significantly $H_{0}$ : “ $X_{n}^{\theta}$ has the uniform distribution" against $H_{1}(\varepsilon)$ : 
" $P\left\{X_{n}^{\theta} \in B o\right\}=L(B o)[1+O b(1) \varepsilon]$ ” if $\sqrt{N} \varepsilon \leq 1 / 10$. Indeed, if $\sqrt{N} \varepsilon=1 / 10$ and $\mathrm{b}=2$, the probability of obtaining $\frac{\sum_{n=1}^{N}\left[1_{[1 / 2,1]}\left(X_{n}^{\theta}\right)-1 / 2\right]}{\sqrt{N / 4}} \geq 2$ is about 0.0466 under $H_{1}(\varepsilon)$ and about 0.0455 under $H_{0}$ : i.e. the probability of rejecting the assumption IID, $H_{0}$, under $H_{1}(\varepsilon)$ is not much bigger than that of rejecting $H_{0}$ if $X_{n}^{\theta}$ is really IID (cf also section 4.3 of [8]).

Then it is no possible to differentiate the IID model and models such that

$$
P\left\{\left(X_{1}^{\theta}, \cdots, X_{N}^{\theta}\right) \in B o\right\}=L(B o)[1+O b(1) \varepsilon] .
$$

\subsubsection{Border of Correct Models}

Now there is a problem : for example, use a realization $x_{n}$ of the IID model, and let $X_{n}^{\theta_{1}}$ be a model checking $P\left\{\left(X_{1}^{\theta_{1}}, \cdots, X_{N}^{\theta_{1}}\right) \in B o\right\}=L(B o)\left[1+O b(1) \varepsilon^{1}\right]$ where

$\varepsilon^{1}$ is small enough but not very small. Let $X_{n}^{\theta_{2}}$ be a model such that

$$
\begin{aligned}
& P\left\{\left(X_{1}^{\theta_{2}}, \cdots, X_{N}^{\theta_{2}}\right) \in B o\right\} \\
& =P\left\{\left(X_{1}^{\theta_{1}}, \cdots, X_{N}^{\theta_{1}}\right) \in B o\right\}[1+O b(1) \varepsilon]^{.}
\end{aligned}
$$

Then, $X_{n}^{\theta_{2}}$ can be not a correct model: it is enough that $\varepsilon^{1}$ is in extreme cases of the possible values of the $\varepsilon$ 's such that

$P\left\{\left(X_{1}^{\theta_{2}}, \cdots, X_{N}^{\theta_{2}}\right) \in B o\right\}=L(B o)[1+O b(1) \varepsilon]$, $\sup _{\text {Во }}(O b(1))=1$, imply that $X_{n}^{\theta}$ is a correct model.

Then, there are models more correct than others. These are models $Y_{n}^{\theta_{0}}$ such that, if $P\left\{\left(Y_{1}^{\theta}, \cdots, Y_{N}^{\theta}\right)\right.$, $Y_{n}^{\theta}$ is also a correct model where $\varepsilon$ is small enough, but not too small. For example, $\varepsilon=1 / 10,1 / 100$ or at worst $\varepsilon=1 / N$ if need be (cf section 5-7 of [7]).

It seems clear that such models exist. For example it is assumed that this is the case when $x_{n}$ is sample of an IID sequence $X_{n}^{\varepsilon}$ and that it is a good realization of $X_{n}^{\varepsilon}$.

On the other hand, we know that it should exist estimates of models (these estimates are easier to calculate in some cases as texts). Then, we can choose as model $Y_{n}^{\theta_{1}}$, the model provided by these estimates : close models will also correct models.

All these points are detailed in [7].

\subsection{Exact IID Model}

Then, generally, if $Y_{n}^{\theta}$ is a correct model such that $T_{q}\left(Y_{n}^{\theta}\right)$ cannot be differentiated with the IID model, one will be able to choose another correct model $Y_{n}^{\theta_{0}}$, close to $Y_{n}^{\theta}$ and such that $T_{q}\left(Y_{n}^{\theta_{0}}\right)$ is exactly the IID model. Indeed one proves easily the following proposition (cf proposition 5-1 of [8]).

Proposition 4.1 One assumes that $m$ is large enough. Let $Y_{n}^{\theta_{c}}$ be a correct model of the sequence $y_{n}$. One assumes that there exists $\varepsilon_{Y}>0$ such that if $Y_{n}^{\theta}$ is a model satisfying, for all Borel set Bo,

$P\left\{\left(X_{1}^{\theta}, \cdots, X_{N}^{\theta}\right) \in B o\right\}=P\left\{\left(X_{1}^{\theta_{c}}, \cdots, X_{N}^{\theta_{c}}\right) \in B o\right\}\left[1+O b(1) \varepsilon_{Y}\right]$, then $Y_{n}^{\theta}$ is a correct model of $y_{n}$.

One assumes also that, for all $\left(k_{1}, \cdots, k_{N}\right)$,

$P\left\{\left\{T_{q}\left(Y_{1}^{\theta_{c}}\right)=k_{1} / 2^{q}\right\} \cap \cdots \cap\left\{T_{q}\left(Y_{N}^{\theta_{c}}\right)=k_{N} / 2^{q}\right\}\right\}$

$=\frac{1}{2^{q N}}\left[1+\varepsilon_{k_{1}, \cdots, k_{N}}(q)\right]$

where $\sup _{k_{1}, \cdots, k_{N}}\left|\varepsilon_{k_{1}, \cdots, k_{N}}(q)\right|=\varepsilon_{X}(q)$. One assumes that $\varepsilon_{X}(q)$ is increasing, that $\varepsilon_{X}(1) \ll \varepsilon_{Y}$ and that there exists $q_{1} \in N^{*}$ such that $\varepsilon_{X}\left(q_{1}\right)$ is small enough.

Then, there exists $q_{0} \in N^{*}$ and a correct model $Y_{n}^{\theta_{0}}$ of the sequence $\left\{y_{n}\right\}_{n=1, \cdots, N}$ such that, for all $\left(k_{1}, \cdots, k_{N}\right)$, $P\left\{\left\{T_{q_{0}}\left(Y_{1}^{\theta_{c}}\right)=k_{1} / 2^{q}\right\} \cap \cdots \cap\left\{T_{q_{0}}\left(Y_{N}^{\theta_{c}}\right)=k_{N} / 2^{q}\right\}\right\}=\frac{1}{2^{q_{0} N}}$

\section{Approximation Theorem}

\subsection{Theorem}

In this section, we assume that $T$ is a Fibonacci congruence and we use Fibonacci function $T_{q}$ in order to build IID sequences.

Theorem 3 We keep the notations 1.3 and 1.4 and notations of section 2.3. Let $\gamma(m)=[2+\varphi(m)]$. We assume $\gamma(m) N K_{0} 2^{q} / m \approx 0$ and $m / K_{0} \gg$. Then, for all Borel set Bo,

$P\left\{\left(X_{1}, \cdots, X_{N}\right) \in B o\right\}=L(B o)\left[1+\frac{\gamma(m) O b^{\prime}(1) N K_{0}}{m / 2^{q}}\right]$.

where $\left|O b^{\prime}(1)\right|$ is increased by a number close to 1.

If $K_{0}$ is not too large, there is no difficulty to choose $m$ and $q$ in such $a$ way that $\varepsilon \leq \gamma(m) 2^{q} N K_{0} / m$ is small enough. Therefore,

$P\left\{\left(X_{1}, \cdots, X_{N}\right) \in B o\right\}=L(B o)[1+O b(1) \varepsilon]$.

\subsection{Proof}

Because, by Section 2.3, the points of $\bar{T}^{-1}(\mathrm{mI})$ are well distributed in $\{0,1, \cdots, m-1\}$, it is easy to prove that the sum of points of $h_{N}^{\prime}\left(\bar{T}^{-1}(\mathrm{mI})\right)$ will be close $\operatorname{card}(m I \cap\{0,1, \cdots, m-1\}) / m$ (e.g. cf Figure 7). Then, we have the following lemma (cf also proposition 6-1 of [7]).

Lemma 5.1 Let $h_{N}$ be the probability density function of $Y \in\{0 / m, 1 / m, \cdots,(m-1) / m\}$, with respect to $\mu_{m}$ : 


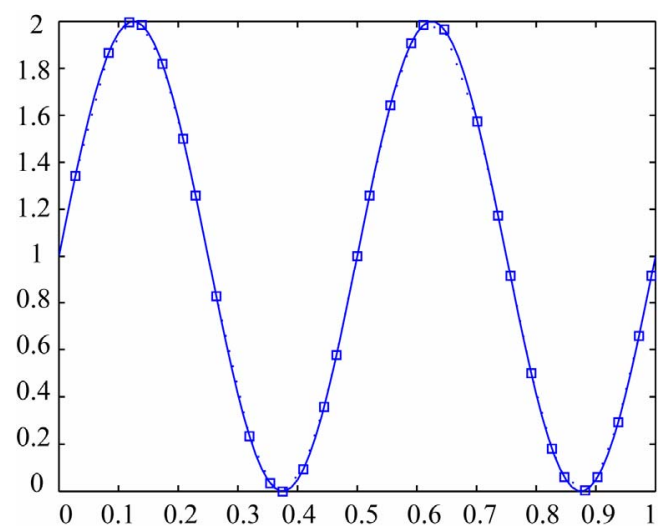

Figure 7. Points of $h_{N}^{\prime}\left(\bar{T}^{-1}\left(m I_{k}\right)\right)$ when $h_{N}^{\prime}(t)=\sin (4 \pi t)+1$.

$\int_{0}^{1} h_{N}(u) \mu_{m}(d u)=1$. Let $h_{N}^{\prime}=\left(1 / c_{0}\right) h_{N} \quad$ such that $\int_{0}^{1} h_{N}^{\prime}(u) \mathrm{d} u=1$.

Let $K_{0}^{\prime} \in R_{+} \quad$ such that $\left|h_{N}(r)-h_{N}\left(r^{\prime}\right)\right| \leq K_{0}^{\prime}\left|r^{\prime}-r\right|$ and $\left|h_{N}^{\prime}(r)-h_{N}^{\prime}\left(r^{\prime}\right)\right| \leq K_{0}^{\prime}\left|r^{\prime}-r\right|$ when $r, r^{\prime} \in[0,1]$. One supposes $2^{q} / m \approx 0$, and $m / K^{\prime}{ }_{0} \gg 1$.

Then, the following equality holds:
$P\left\{\bar{T}(m Y) / m \in I_{k}\right\}=L\left(I_{k}\right)\left[1+\frac{\gamma(m) O b^{\prime}(1) K_{0}^{\prime}}{m / 2^{q}}\right]$,

where $I_{k}=\left[k / 2^{q},(k+1) / 2^{q}\left[, L\left(I_{k}\right)=1 / 2^{q}\right.\right.$.

Then, one can prove theorem 3 . Indeed, by applying lemma 5.1 when $Y$ has for distribution the distribution of the conditional probability of $Y_{\phi(n)}$ given $Y_{\phi(n-1)}=y_{1}^{\prime}, Y_{\phi(n-2)}=y_{2}^{\prime}, \cdots$, we have

$$
\begin{aligned}
& P\left\{\bar{T}(m Y) / m \in I_{k}\right\} \\
& =P\left\{X_{\phi(n)} \in I_{k} \mid Y_{\phi(n-1)}=y_{1}^{\prime}, Y_{\phi(n-2)}=y_{2}^{\prime}, \cdots\right\} \\
& =L\left(I_{k}\right)\left[1+\frac{\gamma(m) O b^{\prime}(1) K_{0}}{m / 2^{q}}\right] .
\end{aligned}
$$

Now, one proves easily that

$$
\begin{aligned}
& P\left\{X_{\phi(n)} \in I_{k} \mid X_{\phi(n-1)}=x_{1}^{\prime}, X_{\phi(n-2)}=x_{2}^{\prime}, \cdots\right\} \\
& =\sum_{y_{s_{1}}^{\prime} \in T_{q}^{-1}\left(x_{1}^{\prime}\right)} \ldots \sum_{y_{s_{N-1}}^{\prime} \in T_{q}^{-1}\left(x_{N-1}^{\prime}\right)} \eta_{y_{s_{1}}^{\prime}, \ldots, y_{s_{N-1}}^{\prime}} \\
& \cdot P\left\{X_{\phi(n)} \in I_{k} \mid Y_{\phi(n-1)}=y_{s_{1}}^{\prime}, Y_{\phi(n-2)}=y_{s_{2}}^{\prime}, \cdots\right\},
\end{aligned}
$$

where

$$
\eta_{y_{s_{1}}^{\prime}, \cdots, y_{s_{N-1}}^{\prime}}=\frac{P\left\{\left\{Y_{\phi(n-1)}=y_{s_{1}}^{\prime}\right\} \cap \cdots \cap\left\{Y_{\phi(n-N+1)}=y_{s_{N-1}}^{\prime}\right\}\right\}}{\sum_{y_{s_{1}}^{\prime} \in T_{q}^{-1}\left(x_{1}^{\prime}\right)} \ldots \sum_{y_{s_{N-1}}^{\prime} \in T_{q}^{-1}\left\{x_{N-1}^{\prime}\right\}} P\left\{\left\{Y_{\phi(n-1)}=y_{s_{1}}^{\prime}\right\} \cap \cdots \cap\left\{Y_{\phi(n-N+1)}=y_{s_{N-1}}^{\prime}\right\}\right\}}
$$

Then, $\sum_{y_{s_{1}}^{\prime} \in T_{q}^{-1}\left(x_{1}^{\prime}\right)} \cdots \sum_{y_{s_{N-1}^{\prime}}^{\prime} \in T_{q}^{-1}\left(x_{N-1}^{\prime}\right)} \eta_{y_{s_{1}}^{\prime}, \cdots, y_{s_{N-1}}^{\prime}}=1$.

We deduce that

$$
\begin{aligned}
& P\left\{X_{\phi(n)} \in I_{k} \mid X_{\phi(n-1)}=x_{1}^{\prime}, X_{\phi(n-2)}=x_{2}^{\prime}, \cdots\right\} \\
& =L\left(I_{k}\right)\left[1+\frac{\gamma(m) O b^{\prime}(1) K_{0}}{m / 2^{q}}\right] .
\end{aligned}
$$

Then, one proves by basic methods (cf proposition 6.2 of [7]) that, for all $I_{k_{1}} \otimes \cdots \otimes I_{k_{N}}$,

$$
\begin{aligned}
& P\left\{\left(X_{1}, \cdots, X_{N}\right) \in I_{k_{1}} \otimes \cdots \otimes I_{k_{N}}\right\} \\
& =\prod_{s=1}^{N}\left(L\left(I_{k_{s}}\right)[1+O b(1) \varepsilon]\right)
\end{aligned}
$$

where $|\varepsilon| \leq \frac{\gamma(m)\left|O b^{\prime}(1)\right| K_{0}}{m / 2^{q}}$. Because

$\gamma(m) N K_{0} 2^{q} / m \approx 0$, we deduce that

$$
\begin{aligned}
& P\left\{\left(X_{1}^{\theta}, \cdots, X_{N}^{\theta}\right) \in I_{k_{1}} \otimes \cdots \otimes I_{k_{N}}\right\} \\
& =\frac{1}{2^{N q}}\left[1+\frac{\gamma(m) O b^{\prime}(1) N K_{0}}{m / 2^{q}}\right] .
\end{aligned}
$$

Now, we deduce that, for all Borel set

$$
\begin{aligned}
& B o \subset\left\{0 / 2^{q}, \cdots,\left(2^{q}-1\right) / 2^{q}\right\}^{N}, \\
& P\left\{\left(X_{1}, \cdots, X_{N}\right) \in B o\right\}=L(B o)\left[1+\frac{\gamma(m) O b^{\prime}(1) N K_{0}}{m / 2^{q}}\right] .
\end{aligned}
$$

\section{Choice of Random Noises}

\subsection{Use of Texts}

Now, we suppose that we use sequences $y_{n}^{\prime}$ and $y^{\prime \prime}{ }_{n} \in\{0 / m, 1 / m, \cdots,(m-1) / m\}, n=1,2, \cdots, N$, obtained from independent texts. In order to reduce $K_{0}$ we add modulo $\mathrm{m}$ a text and a text written backward:

$y_{n}=\left[\overline{y_{n}^{\prime}+y^{\prime \prime}{ }_{N-n+1}+\operatorname{rand}_{0}(n)}\right] / m$ where $\operatorname{rand}_{0}(n)$ is

pseudo-random sequences which have good empirical independence assumptions for $\mathrm{p}$ successive pseudo random numbers when $p \geq 3$. In an obvious way, the texts are realizations of sequences of random variables : for example, one can take as model, the set of the possible texts provided with the uniform probability

As a matter of fact, we add $\operatorname{rand}_{0}(n)$ to have 
sequences $y_{n}$ which have a good randomness (cf [9], or chapter 3 of [6]).

In particular, a priori, " $P\left\{Y_{n}=y\right\}$ is not too different from $1 / \mathrm{m}$ " is a reasonable assumption. Moreover, $\left(y_{n}, y_{n+1}, y_{n+2}\right)$ has a empirical distribution close to independence and texts behaves as Q-dependent sequences (cf [6]). Then, for all permutation $\phi$, a three-dimensional model $\left(Y_{\phi(n)}, Y_{\phi(n+1)}, Y_{\phi(n+2)}\right)$ with a continuous density and a Lipschitz coefficient not too big will be a good model. By the same way,

$$
P\left\{Y_{\phi(n)}=y \mid Y_{\phi(n-1)}=y_{1}^{\prime}, Y_{\phi(n-2)}=y_{2}^{\prime}, \cdots\right\} \text { is not too }
$$

different of $P\left\{Y_{n}=y\right\}$ which is not too different from $1 / \mathrm{m}$ (cf [7]). In this case, one can prove that generally $K_{0}$ is small cf [7].

On the other hand, to increase $K_{0}$ a good way is to use the Central Limit theorem. In fact one can combine the two methods : $\mathrm{cf}[7]$.

\subsection{Example}

Now it may be necessary to do some transformations to get the $m y_{n}^{\prime} \in\{0,1, \cdots, m-1\}$ in the case where the letters and symbols are provided by sequences $\mathrm{a}(j)$, $j=1,2, \cdots, N_{3}, a(j) \in\{0,1, \cdots, 255\}, \quad j=1,2, \cdots, N_{3}$.

One sets $N_{0}=\left\lfloor N_{3} / r_{1}\right\rfloor$. We choose two consecutive elements $\mathrm{a}$ and $\mathrm{m}$ of the Fibonacci sequence : $\mathrm{m}$ can be chosen with respect to $N_{0}$. Then, we choose $r_{1}$ such that $a<32^{r_{1}} \leq m$.

1) We set $c(j)=\overline{a(j)} \bmod \kappa=32$

2) We set $d(n)=\sum_{r=1}^{r_{1}} c\left(r_{1}(n-1)+r\right) \kappa^{r-1}$ for $j=1,2, \cdots, N_{0}$.

3) We set $y_{n}^{\prime}=\left\lfloor d(n) m / \kappa^{r_{1}}\right\rfloor / m$ for $j=1,2, \cdots, N_{0}$.

By using this technique, we have created real IIID sequences $x_{n}$. We have used a sequence $c(j)$ with $N_{3}=20,000,000$. This sequence was obtained from dictionary, encyclopedia, and Bible. We choose $r_{1}=20$, $a<4 * 10^{36} \leq m, q=70$. Then $N_{0}=10^{6}$. Then, we have estimated $K_{0}=0.01$. In order to avoid any error we have choose $K_{0}=10^{4}$ in the building of $x_{n}$.

We have tested the sequence $x_{n}$. We have used the classical Diehard tests (cf [1] [2]) and the higher order correlation coefficients (cf [10]). Results are in accordance with what we waited: the hypothesis "randomness" is accepted by all these tests (cf [7]).

One can can download this sequence in [11]

\section{Conclusion: Building of Random Sequence}

By theorem 3 one can find models correct $Y_{n}^{\theta}$ such that $P\left\{\left(X_{1}^{\theta}, \cdots, X_{N}^{\theta}\right) \in B o\right\}=L(B o)[1+O b(1) \varepsilon]$ where $\varepsilon$ is small if $K_{0}$ is not too large. Now it is possible to build such sequences concretely, for example by using texts studied in section 6 . In this case, coefficient $K_{0}$ depend on the choice of $\mathrm{m}$, i.e. of $r_{1}$. But, $K_{0}$ increases very little when $r_{1}$ increases. Even, in some cases, it seems that it decreases. Then, at most $2^{q} / m$ decreases much more quickly than $K_{0}$ increases.

So by taking $\mathrm{m}$ large enough and by choosing well $\mathrm{q}$, we found $\varepsilon$ small enough in a way that there exists correct models which checks the conditions of proposition 4.1. Then, there exists $m$ sufficiently large and q sufficiently small and a correct model

$Y_{n}^{\theta_{0}} \in\{0 / m, 1 / m, \cdots,(m-1) / m\}$ such that $T_{q}\left(Y_{n}^{\theta_{0}}\right)$ is the IID model.

Then, this result show that one can build sequences $x_{n}$ such that the model IID is a correct model of $x_{n}$.

That means that $x_{n}$ behaves like any IID sample: a priori, $x_{n}$ can check not the properties which one expects from a IID sample like certain tests, but that occurs only with a probability equal to that of any IID sample.

By this method, we therefore have a mean to value the technique used by Marsaglia to create its CD-ROM. We arrive in fact to prove mathematically that the sequence obtained can be regarded a priori as random, what Marsaglia did not.

Remark 7.1 One might wonder if the sequence built adding text and pseudo-random sequences is not an IID sequence. It is a similar hypothesis which Marsaglia does when he built its CD-Rom. This also corresponds to results of [9]. But in fact, nothing is proved.

It is maybe possible to prove it but that seems complicated. Finally, it is much easier to apply the functions $T_{q}$ : in this case, it requires only that $K_{0}$ is not too big. It's an hypothesis much simpler to be verified and it does not require many efforts in some cases. That is why we choose to build IID sequences using this technique.

\section{References}

[1] D. E. Knuth, "The Art of Computer Programming," 3rd Edition, Addison-Wesley, Boston, 1998.

[2] J. Gentle, "Random Number Generation and Monte Carlo Method," Springer 13, 1984, pp. 61-81.

[3] A. Menezes, P. Van Oorschot, S. Vanstone, "Handbook of Applied Cryptography," CRC Press, London, 1996. doi:10.1201/9781439821916

[4] R. Blacher, "Solution Complete Au Probleme des Nombres Aleatoires," 2004.

http://www.agro-montpellier.fr/sfds/CD/textes/blacher1.p df

[5] U. Dieter, "Statistical Interdependence of Pseudo Random numbers Generated by the Linear Congruential 
Method," In: S. K. Zaremba, Ed., Applications of Number Theory to Numerical Analysis, Academic Press, New York, 1972, pp. 287-317.

[6] R. Blacher, "A Perfect Random Number Generator," Rapport de Recherche LJK Universite de Grenoble, 2009. http://hal.archives-ouvertes.fr/hal-00426555/fr/

[7] R. Blacher, "Fibonacci Congruences and Applications," Rapport de Recherche LJK Universite de Grenoble, 2011. http://hal.archives-ouvertes.fr/hal-00587108/fr/.

[8] R. Blacher, "Correct models," Rapport de Recherche LJK Universite de Grenoble, 2010.

http:/hal.archives-ouvertes.fr/hal-00521529/fr/
[9] L. Y. Deng and E. O. George, "Some Characterizations of the Uniform Distribution with Applications to Random Number Generation," Annals of the Institute of Statistical Mathematics, Vol. 44, No. 2, 1992, pp. 379-385. doi:10.1007/BF00058647

[10] R. Blacher, "Higher Order Correlation Coefficients," Statistics, Vol. 25, No. 1, 1993, pp. 1-15. doi:10.1080/02331889308802427

[11] R. Blacher, File of random Number, 2009. http://www-ljk.imag.fr/membres/Rene.Blacher/GEAL/no de3.html. 